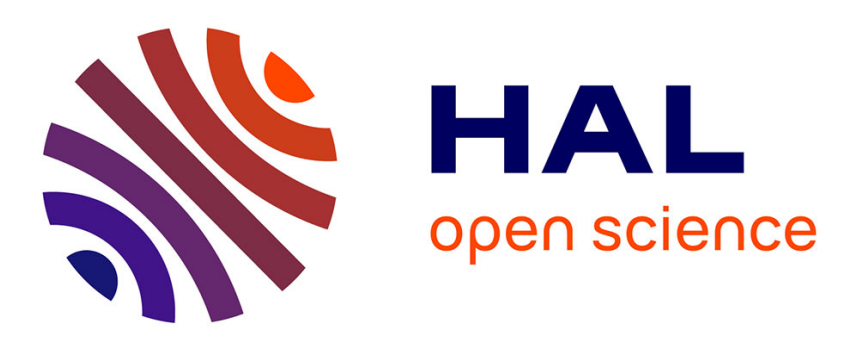

\title{
A one-pot chemoselective synthesis of secondary amines by using a biomimetic electrocatalytic system
}

\author{
Martine Largeron
}

\section{To cite this version:}

Martine Largeron. A one-pot chemoselective synthesis of secondary amines by using a biomimetic electrocatalytic system. Electrochimica Acta, 2009, 54 (22), pp.5109-5115. 10.1016/j.electacta.2009.01.005 . hal-02384927

\section{HAL Id: hal-02384927 \\ https://hal.science/hal-02384927}

Submitted on 12 Jan 2021

HAL is a multi-disciplinary open access archive for the deposit and dissemination of scientific research documents, whether they are published or not. The documents may come from teaching and research institutions in France or abroad, or from public or private research centers.
L'archive ouverte pluridisciplinaire HAL, est destinée au dépôt et à la diffusion de documents scientifiques de niveau recherche, publiés ou non, émanant des établissements d'enseignement et de recherche français ou étrangers, des laboratoires publics ou privés. 


\title{
A One-Pot Chemoselective Synthesis of Secondary Amines by Using a Biomimetic Electrocatalytic System
}

\author{
Martine Largeron*,1 \\ UMR CNRS 8638, Synthèse et Structure de Molécules d'Intérêt Pharmacologique, Université Paris \\ Descartes, Faculté des Sciences Pharmaceutiques et Biologiques, 4 Avenue de l'Observatoire, 75270 \\ Paris Cedex 06, France
}

\begin{abstract}
A one-pot electrochemically induced oxidation-imine formation-reduction route to secondary amines is described in detail. The key step of the process consists of the $o$ iminoquinone-mediated chemoselective catalytic oxidation of a primary aliphatic amine substrate, in the presence of a second amine used as the alkylating agent. Through the examination of the scope of the reaction by systematically varying both amine substrate and amine alkylating agent, it can be shown that this reaction sequence, leaving ammonia as the sole by-product, allows the rapid synthesis of various secondary amines in moderate to good yields. This process, that highlights the pre-eminent green advantages of electrochemical synthesis, especially the utilization of electricity as energy instead of chemical reagents, high atom economy as well as ambient temperature and pressure, could be a mild alternative to already reported synthetic methods.
\end{abstract}

Keywords : amine, chemoselectivity, electrocatalysis, indirect anodic oxidation, one-pot sequence

* fax: 3301440735 88, E-mail:martine.largeron@parisdescartes.fr

${ }^{1}$ ISE member 


\section{Introduction}

Because of their potential as useful synthetic intermediates and as robust pharmacophores, the synthesis of secondary amines has long attracted much attention and is still an active area of research [1]. In the past decades, conventional methods has been extensively utilized for the synthesis of secondary amines: the direct $\mathrm{N}$-alkylation with an alkyl halide or similar alkylating agent, and the reductive amination with carbonyl compounds. However, overalkylation may compromise the yield of the reaction, while the use of toxic alkyl halides or strong reducing reagents, as well as the production of equimolar amounts of wasteful salts as by-products, is undesirable from an environmental viewpoint.

An attractive combination of both methods employs alcohols as the starting materials, which undergo a net loss of hydrogen to provide carbonyl compounds that form an imine which is reduced to an amine. Accordingly, the performance of an in situ oxidation-imine formation-reduction sequence using manganese dioxide in combination with sodium borohydride or polymer-supported cyanoborohydride, has been reported for the conversion of activated alcohols (benzylic, allylic and propargylic) into secondary amines [2-4]. Although redox processes are well-known in biological systems, a chemical oxidant and reductant can however coexist in only rare circumstances in conventional organic chemistry. This is probably the reason why little attention has been devoted to such metal-free one-pot sequences which are attractive because avoiding the use of toxic alkylating halides whereas alcohols are readily available substrates. Furthermore, the intermediate aldehydes and imines do not require isolation. This is particularly useful in cases where the intermediate is unstable or difficult to handle (e.g. toxic, volatile, or prone to polymerization).

More recently, considerable efforts have been directed towards the development of related metal-catalyzed sequence and several catalyst systems have been described for the N- 
alkylation of amines with alcohols, using ruthenium and other transition metal catalyst [5-13]. In these processes, the catalyst has high activity for both the dehydrogenation of alcohol to aldehyde and the hydrogenation of the resulting imine to amine. Interestingly, there is no net hydrogen gain and loss during the reaction sequence, while water is produced as the only byproduct, so that high atom economy can be realized. A related approach consists of the Nalkylation of amines with amines instead of alcohols. A noteworthy example is the selective ruthenium-catalyzed $\mathrm{N}$-alkylation of aryl amines using aliphatic amines which produces the corresponding $N$-alkyl-aryl amines, in good yields, leaving ammonia as the only by-product without extrusion of water [14-16].

Related oxidation-imine formation-reduction route to secondary amines could also be achieved electrolytically, but the fact that aliphatic primary amines are oxidized at relatively high anodic potentials $(>+1.5 \mathrm{~V}$ vs $\mathrm{SCE})$ to yield unstable cation radicals that rapidly deprotonate and attach to the electrode surface, does not make it possible directly [17-19].

Recently, we reported the high catalytic performance of the redox mediator 3,4iminoquinone $\mathbf{1}_{\mathbf{o x}}$ in the chemoselective indirect electrochemical oxidation of primary aliphatic amines, under metal-free conditions $[20,21]$. The mechanism was very close to the ionic pyridoxal-like transamination process reported for amine oxidase cofactors. The formation of a highly reactive Schiff base cyclic transition state (step 3, Scheme 1), which allowed the activation of the imine function for further nucleophilic attack by the amine, constituted the driving force for the overall transamination mechanism [21]. The catalytic cycle produced the reduced catalyst $\mathbf{1}_{\text {red }}$ and an unstable alkylimine as the product of amine oxidation (step 4, Scheme 1), at room temperature, leaving ammonia as the sole by-product. These conditions were particularly favorable for using the imine in situ for further reactions. In particular, by coupling the $\mathbf{1}_{\mathbf{o x}}$-mediated catalytic process with an electrochemical reduction of the extruded alkylimine (step 5, Scheme 1), a secondary amine could be then prepared. 
Unfortunately, the yield of the reaction could not exceed $50 \%$, because two molecules of primary amine (steps 2 and 4, Scheme 1) are required to produce one molecule of alkylimine. Furthermore, secondary amines possessing different substituents on both sides of the nitrogen atom could not be synthesized by this way. To circumvent these limitations, we sought to modify our initial electrocatalytic procedure. Herein, we describe in detail a one-pot metalfree $\mathbf{1}_{\text {ox }}$-mediated oxidation-imine formation-reduction sequence for the highly atomeconomical chemoselective $\mathrm{N}$-alkylation of primary aliphatic amines with amines, under mild conditions.

\section{Experimental}

Chemicals were commercial products of the highest available purity and were used as supplied. Reduced catalyst $\mathbf{1}_{\text {red }}$ was synthesized as earlier reported [22]. All apparatus, cells and electrodes were identical with those described previously [21]. ${ }^{1} \mathrm{H}$ NMR and ${ }^{13} \mathrm{C}$ NMR spectra were recorded on a Brucker AC 300 spectrometer operating at $300 \mathrm{MHz}$ for ${ }^{1} \mathrm{H}$ observations. Chemical shifts (in $\mathrm{ppm}$ ) are relative to internal tetramethylsilane. The measurements were carried out using the standard pulse sequences. The carbon type [methyl, methylene, methide or quaternary (noted Cq)] was determined by DEPT experiments. The identity of known secondary amines $\mathbf{A}$ and $\mathbf{C}$ (Scheme 3) as well as that of known vicinal diamines B (Scheme 3) was confirmed by ${ }^{1} \mathrm{H}$ and ${ }^{13} \mathrm{C}$ NMR after comparison with previously reported data: dibenzylamine [23], $N$-benzyl- $N$-cyclopropylmethylamine (entry 1, Table 1) [24]; $N$-benzyl- $N$-cyclohexylmethylamine (entry 2 , Table $\quad 1) \quad[25] ; \quad N$-benzyl- $N$ cyclohexylamine (entry 3, Table 1) [26]; $N$-benzyl(3,3-dimethyl)butylamine (entry 5, Table 1) [27]; $N$-benzylhexylamine (entry 6, Table 1) [27,28]; $N$-benzyl-1-phenyl-2-propylamine (entry 7, Table 1) [29]; N,N-di(2-methoxyethyl)-1,2-bisphenylethane-1,2-diamine (entry 8, 
Table 1) [30], benzyl(2,2-dimethoxyethyl)amine (entry 9, Table 1) [31]; 2benzylaminoethanol (entry 10, Table 1) [32]; $N, N$-bis-( $p$-fluorobenzyl)amine (entry 1, Table 2), $N, N$-bis-( $p$-methylbenzyl)amine (entry 2, Table 2$)$ and $N, N$-bis-( $p$-methoxybenzyl)amine (entry 3, Table 2) [33]; $N$-(4-methoxybenzyl)-3-phenylpropylamine (entry 3, Table 2) [34]; $N, N$-bis-(benzo-1,3-dioxol-5-ylmethyl)amine (entry 4, Table 2) [35]; N,N-bis-naphtalen-2-ylmethylamine (entry 5, Table 2) [36]; $N$-(3-phenylpropyl)-2-pyridinemethanamine (entry 6, Table 2) [37]; N-(2-pyridinylmethyl)-2-pyridinemethanamine (entry 6, Table 2) [38]; $\mathrm{N}$-(3phenylpropyl)-3-pyridinemethanamine (entry 7, Table 2) [39]; N-(3-pyridinylmethyl)-3pyridinemethanamine (entry 7, table 2) [38].

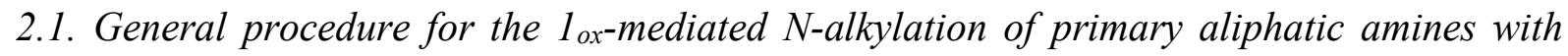
amines

Controlled-potential electrolysis was carried out in a cylindrical, three-electrode divided cell (9 $\mathrm{cm}$ diameter), using an electronic potentiostat. In the main compartment, a platinum grid $\left(90 \mathrm{~cm}^{2}\right.$ area) served as the anode (working electrode). A platinum sheet was placed in the concentric cathodic compartment (counter electrode), which was separated from the main compartment with a glass frit. The reference electrode, to which all quoted potentials are referred, was an aqueous saturated calomel electrode (SCE). The electrolyte solution $(0.1$ mol $\mathrm{L}^{-1}$ lithium perchlorate in methanol) was poured into the anodic and the cathodic compartments, as well as into the glass tube that contained the SCE electrode. Reduced catalyst $\mathbf{1}_{\text {red }}(0.1 \mathrm{mmol})$ ( $4 \mathrm{~mol} \%$ relative to primary aliphatic amine substrate), primary aliphatic amine substrate $(2.5 \mathrm{mmol})$, together with a slight excess of alkylating amine $(3.75$ mmol) were added to the solution in the main compartment $(250 \mathrm{~mL})$, and the resulting solution was then oxidized under nitrogen, at room temperature, at $+0.6 \mathrm{~V}$ vs SCE (initial current $60-70 \mathrm{~mA})$. After exhaustive anodic electrolysis (3h), the platinum anode was 
replaced by a mercury pool whose the potential was fixed at $-1.6 \mathrm{~V}$ vs SCE. After $1 \mathrm{~h}$, when a negligible value of the current was recorded indicating the end of the electrochemical reduction, the electrolysis solution was poured into an aqueous $\mathrm{HCl}$ solution $\left(0.5 \mathrm{~mol} \mathrm{~L}^{-1}, 15\right.$ $\mathrm{mL}$ ) and $\mathrm{MeOH}$ was removed under reduced pressure. The resulting solution was treated with an aqueous $\mathrm{K}_{2} \mathrm{CO}_{3}$ solution $(0.5 \mathrm{~mol} \mathrm{~L}-1,15 \mathrm{~mL})$ and extracted with $\mathrm{CH}_{2} \mathrm{CL}_{2}(100 \mathrm{~mL})$. The organic layer was then dried over $\mathrm{MgSO}_{4}$ and the solvent removed under reduced pressure at $30^{\circ} \mathrm{C}$. Flash column chromatography of the residue on silica allowed the purification of the expected secondary amine.

\subsection{Structures for which no previously spectral data is available}

4-(N-benzyl-2-aminoethyl)morpholine. (entry 4, Table 1) (previously described as the dihydrochloride salt) [40]. Colorless oil. ${ }^{1} \mathrm{H}$ NMR (300 $\left.\mathrm{MHz}, \mathrm{CDCl}_{3}, 25^{\circ} \mathrm{C}, \mathrm{TMS}\right): \delta=2.20$ (s, $1 \mathrm{H} ; \mathrm{NH}), 2.40\left(\mathrm{~m}, 4 \mathrm{H} ; \mathrm{CH}_{2}\right), 2.50\left(\mathrm{t}, J=6 \mathrm{~Hz}, 2 \mathrm{H} ; \mathrm{CH}_{2}\right), 2.70\left(\mathrm{t}, J=6 \mathrm{~Hz}, 2 \mathrm{H} ; \mathrm{CH}_{2}\right), 3.70$ (m, 4H; $\left.\mathrm{CH}_{2}\right), 3.80\left(\mathrm{~s}, 2 \mathrm{H} ; \mathrm{CH}_{2}\right), 7.25-7.35(\mathrm{~m}, 5 \mathrm{H}, \mathrm{CH}) ;{ }^{13} \mathrm{C} \mathrm{NMR}\left(75 \mathrm{MHz}, \mathrm{CDCl}_{3}, 25^{\circ} \mathrm{C}\right.$, TMS $): \delta=45.1\left(\mathrm{CH}_{2}\right), 53.7\left(\mathrm{CH}_{2}\right), 53.9\left(\mathrm{CH}_{2}\right), 58.1\left(\mathrm{CH}_{2}\right), 67.0\left(\mathrm{CH}_{2}\right), 127.0(\mathrm{CH}), 128.1$ (CH), $128.4(\mathrm{CH}), 140.2(\mathrm{Cq}) ; \mathrm{MS}(\mathrm{ES}+) \mathrm{m} / z 221\left(\mathrm{MH}^{+}\right)$.

N-benzyl-2-methoxyethylamine. (entry 8, Table 1) (previously described as the oxalate salt [41]. Colorless oil. ${ }^{1} \mathrm{H}$ NMR (300 MHz, $\left.\mathrm{CDCl}_{3}, 25^{\circ} \mathrm{C}, \mathrm{TMS}\right): \delta=1.90$ (s, 1H; NH), 2.80 (t, $J$ $\left.=5 \mathrm{~Hz}, 2 \mathrm{H} ; \mathrm{CH}_{2}\right), 3.35\left(\mathrm{~s}, 3 \mathrm{H}, \mathrm{CH}_{3}\right), 3.50\left(\mathrm{t}, \mathrm{J}=5 \mathrm{~Hz}, 2 \mathrm{H} ; \mathrm{CH}_{2}\right), 3.80\left(\mathrm{~s}, 2 \mathrm{H} ; \mathrm{CH}_{2}\right), 7.25-7.35$ $(\mathrm{m}, 5 \mathrm{H}, \mathrm{CH}) ;{ }^{13} \mathrm{C} \mathrm{NMR}\left(75 \mathrm{MHz}, \mathrm{CDCl}_{3}, 25^{\circ} \mathrm{C}, \mathrm{TMS}\right): \delta=48.8\left(\mathrm{CH}_{2}\right), 54.0\left(\mathrm{CH}_{2}\right), 58.8$ $\left(\mathrm{CH}_{3}\right), 72.0\left(\mathrm{CH}_{2}\right), 126.9(\mathrm{CH}), 128.2(\mathrm{CH}), 128.4(\mathrm{CH}), 140.3(\mathrm{Cq})$; MS (ES+) m/z 166 $\left(\mathrm{MH}^{+}\right)$. 
N,N-di(cyclohexylmethyl)-1,2-bisphenylethane-1,2-diamine (entry 2, Table 1).

Meso form: Colorless solid: ${ }^{1} \mathrm{H}$ NMR $\left(300 \mathrm{MHz}, \mathrm{CDCl}_{3}, 25^{\circ} \mathrm{C}, \mathrm{TMS}\right): \delta=0.50-1.70(\mathrm{~m}$, 22H; $\mathrm{CH}_{2}$ and $\mathrm{CH}$ (cyclohexyl), $\left.\mathrm{NH}\right), 2.10$ (m, 4H; $\left.\mathrm{CH}_{2}\right), 3.68$ (s, 2H; $\left.\mathrm{CH}\right), 7.29-7.35$ (m, $10 \mathrm{H} ; \mathrm{CH}) ;{ }^{13} \mathrm{C}$ NMR $\left(75 \mathrm{MHz}, \mathrm{CDCl}_{3}, 25^{\circ} \mathrm{C}, \mathrm{TMS}\right): \delta=26.0\left(\mathrm{CH}_{2}\right), 26.05\left(\mathrm{CH}_{2}\right), 26.7$ $\left(\mathrm{CH}_{2}\right), 31.1\left(\mathrm{CH}_{2}\right), 31.2\left(\mathrm{CH}_{2}\right), 37.6(\mathrm{CH}), 54.2\left(\mathrm{CH}_{2}\right), 68.6(\mathrm{CH}), 127.3(\mathrm{CH}), 128.2(\mathrm{CH})$, $128.4(\mathrm{CH}), 141.6(\mathrm{Cq})$; MS (ES+) $m / z 405\left(\mathrm{MH}^{+}\right)$; Anal. Cacld for $\mathrm{C}_{28} \mathrm{H}_{40} \mathrm{~N}_{2}$ : C, 83.17; H, 9.90; N, 6.93\%. Found: C, 83.04; H, 9.92; N, 6.88\%.

$d$,l-form: colorless oil: ${ }^{1} \mathrm{H}$ NMR $\left(300 \mathrm{MHz}, \mathrm{CDCl}_{3}, 25^{\circ} \mathrm{C}, \mathrm{TMS}\right): \delta=0.70-2.20(\mathrm{~m}, 24 \mathrm{H}$;

$\mathrm{CH}_{2}$ and $\mathrm{CH}$ (cyclohexyl), $\left.\mathrm{NH}\right), 2.30\left(\mathrm{~m}, 4 \mathrm{H} ; \mathrm{CH}_{2}\right), 3.61(\mathrm{~s}, 2 \mathrm{H} ; \mathrm{CH}), 7.00-7.25(\mathrm{~m}, 10 \mathrm{H}$; $\mathrm{CH}) ;{ }^{13} \mathrm{C}$ NMR $\left(75 \mathrm{MHz}, \mathrm{CDCl}_{3}, 25^{\circ} \mathrm{C}, \mathrm{TMS}\right): \delta=26.2\left(\mathrm{CH}_{2}\right), 26.8\left(\mathrm{CH}_{2}\right), 31.4\left(\mathrm{CH}_{2}\right), 31.5$ $\left(\mathrm{CH}_{2}\right), 38.2(\mathrm{CH}), 54.6\left(\mathrm{CH}_{2}\right), 69.7(\mathrm{CH}), 126.7(\mathrm{CH}), 127.8(\mathrm{CH}), 127.9(\mathrm{CH}), 142.0(\mathrm{Cq})$; MS (ES+) $m / z 405\left(\mathrm{MH}^{+}\right)$.

N,N-di(2,2-dimethoxyethyl)-1,2-bisphenylethane-1,2-diamine (entry 9, table 1)

Meso form: pale yellow solid: ${ }^{1} \mathrm{H}$ NMR $\left(300 \mathrm{MHz}, \mathrm{CDCl}_{3}, 25^{\circ} \mathrm{C}\right.$, TMS) : $\delta=1.70$ (bs, $2 \mathrm{H}$; $\mathrm{NH}), 2.44\left(\mathrm{~m}, 4 \mathrm{H} ; \mathrm{CH}_{2}\right), 3.14\left(\mathrm{~s}, 6 \mathrm{H} ; \mathrm{CH}_{3}\right), 3.19\left(\mathrm{~s}, 6 \mathrm{H} ; \mathrm{CH}_{3}\right), 3.73(\mathrm{~s}, 2 \mathrm{H} ; \mathrm{CH}), 4.30$ (t, J=6 $\mathrm{Hz}, 2 \mathrm{H} ; \mathrm{CH}), 7.29-7.37(\mathrm{~m}, 10 \mathrm{H} ; \mathrm{CH}) ;{ }^{13} \mathrm{C} \mathrm{NMR}\left(75 \mathrm{MHz}, \mathrm{CDCl}_{3}, 25^{\circ} \mathrm{C}, \mathrm{TMS}\right): \delta=48.6$ $\left(\mathrm{CH}_{2}\right), 53.0\left(\mathrm{CH}_{3}\right), 53.7\left(\mathrm{CH}_{3}\right), 68.6(\mathrm{CH}), 103.2(\mathrm{CH}), 127.7(\mathrm{CH}), 128.35(\mathrm{CH}), 128.4(\mathrm{CH})$, $140.8(\mathrm{Cq})$; MS (ES+) m/z $389\left(\mathrm{MH}^{+}\right)$; Anal. Cacld for $\mathrm{C}_{22} \mathrm{H}_{32} \mathrm{~N}_{2} \mathrm{O}_{4}: \mathrm{C}$, 68.04; $\mathrm{H}, 8.25 ; \mathrm{N}$, 7.22\%. Found: C, 68.00; H, 8.21; N, 7.19\%.

$d, l$-form: pale yellow oil: ${ }^{1} \mathrm{H}$ NMR $\left(300 \mathrm{MHz}, \mathrm{CDCl}_{3}, 25^{\circ} \mathrm{C}, \mathrm{TMS}\right): \delta=1.90$ (bs, $2 \mathrm{H}$; $\mathrm{NH}$ ), $2.56\left(\mathrm{~m}, 4 \mathrm{H} ; \mathrm{CH}_{2}\right), 3.28\left(\mathrm{~s}, 6 \mathrm{H} ; \mathrm{CH}_{3}\right), 3.32\left(\mathrm{~s}, 6 \mathrm{H} ; \mathrm{CH}_{3}\right), 3.61(\mathrm{~s}, 2 \mathrm{H} ; \mathrm{CH}), 4.45(\mathrm{t}, J=6 \mathrm{~Hz}$, 2H; $\mathrm{CH}), 7.00-7.18(\mathrm{~m}, 10 \mathrm{H} ; \mathrm{CH}) ;{ }^{13} \mathrm{C} \mathrm{NMR}\left(75 \mathrm{MHz}, \mathrm{CDCl}_{3}, 25^{\circ} \mathrm{C}, \mathrm{TMS}\right): \delta=48.9\left(\mathrm{CH}_{2}\right)$, $53.3\left(\mathrm{CH}_{3}\right), 53.7\left(\mathrm{CH}_{3}\right), 69.3(\mathrm{CH}), 103.7(\mathrm{CH}), 126.9(\mathrm{CH}), 127.8(\mathrm{CH}), 127.9(\mathrm{CH}), 141.0$ (Cq); MS (ES+) $m / z 389\left(\mathrm{MH}^{+}\right)$. 
N-(4-Fluorobenzyl)-3-phenylpropylamine (entry 1, Table 2). Colorless oil. ${ }^{1} \mathrm{H}$ NMR (300 $\left.\mathrm{MHz}, \mathrm{CDCl}_{3}, 25^{\circ} \mathrm{C}, \mathrm{TMS}\right): \delta=1.56(\mathrm{~s}, 1 \mathrm{H} ; \mathrm{NH}), 1.88$ (quintet, $\left.J=7 \mathrm{~Hz}, 2 \mathrm{H} ; \mathrm{CH}_{2}\right), 2.70(\mathrm{~m}$, 4H, $\left.\mathrm{CH}_{2}\right), 3.78\left(\mathrm{~s}, 2 \mathrm{H} ; \mathrm{CH}_{2}\right), 7.05(\mathrm{~m}, 2 \mathrm{H} ; \mathrm{CH}), 7.21-7.33(\mathrm{~m}, 7 \mathrm{H} ; \mathrm{CH}) ;{ }^{13} \mathrm{C} \mathrm{NMR}(75 \mathrm{MHz}$, $\left.\mathrm{CDCl}_{3}, 25^{\circ} \mathrm{C}, \mathrm{TMS}\right): \delta=31.7\left(\mathrm{CH}_{2}\right), 33.6\left(\mathrm{CH}_{2}\right), 48.8\left(\mathrm{CH}_{2}\right), 53.2\left(\mathrm{CH}_{2}\right), 115.2(\mathrm{CH}), 125.8$ $(\mathrm{CH}), 128.4(\mathrm{CH}), 129.7(\mathrm{CH}), 136.1(\mathrm{Cq}), 142.1(\mathrm{Cq}), 161.5(\mathrm{Cq}) ; \mathrm{MS}(\mathrm{ES}+) \mathrm{m} / z 244$ $\left(\mathrm{MH}^{+}\right)$; Anal. Cacld for $\mathrm{C}_{16} \mathrm{H}_{18} \mathrm{FN}$ : C, 79.01; H, 7.41; N, 5.76. Found: C, 78.85; H, 7.43; N, 5.70.

N-(4-methylbenzyl)-3-phenylpropylamine (entry 2, Table 2). Colorless oil. ${ }^{1} \mathrm{H}$ NMR (300 $\mathrm{MHz}, \mathrm{CDCl}_{3}, 25^{\circ} \mathrm{C}, \mathrm{TMS}$ ) : $\delta=1.58(\mathrm{~s}, 1 \mathrm{H} ; \mathrm{NH}), 1.88$ (quintet, $J=7 \mathrm{~Hz}, 2 \mathrm{H} ; \mathrm{CH}_{2}$ ), 2.38 (s, 3H; $\left.\mathrm{CH}_{3}\right), 2.71\left(\mathrm{~m}, 4 \mathrm{H} ; \mathrm{CH}_{2}\right), 3.79\left(\mathrm{~s}, 2 \mathrm{H} ; \mathrm{CH}_{2}\right), 7.17-7.32(\mathrm{~m}, 9 \mathrm{H} ; \mathrm{CH}) ;{ }^{13} \mathrm{C} \mathrm{NMR}(75 \mathrm{MHz}$, $\left.\mathrm{CDCl}_{3}, 25^{\circ} \mathrm{C}, \mathrm{TMS}\right): \delta=21.1\left(\mathrm{CH}_{3}\right), 31.7\left(\mathrm{CH}_{2}\right), 33.7\left(\mathrm{CH}_{2}\right), 48.9\left(\mathrm{CH}_{2}\right), 53.7\left(\mathrm{CH}_{2}\right), 125.8$ $(\mathrm{CH}), 128.1(\mathrm{CH}), 128.3(\mathrm{CH}), 128.4(\mathrm{CH}), 129.1(\mathrm{CH}), 136.5(\mathrm{Cq}), 137.4(\mathrm{Cq}), 142.2(\mathrm{Cq})$; MS (ES+) $m / z 240\left(\mathrm{MH}^{+}\right)$; Anal. Cacld for $\mathrm{C}_{17} \mathrm{H}_{21} \mathrm{~N}$ : C, 85.35; H, 8.78; N, 5.86. Found: C, 85.30; H, 8.76; N, 5.82 .

$\mathrm{N}$-(3,4-methylenedioxybenzyl)phenylpropylamine (entry 4, Table 2). Colorless oil. ${ }^{1} \mathrm{H}$ NMR $\left(300 \mathrm{MHz}, \mathrm{CDCl}_{3}, 25^{\circ} \mathrm{C}, \mathrm{TMS}\right): \delta=1.40(\mathrm{~s}, 1 \mathrm{H} ; \mathrm{NH}), 1.88$ (quintet, $J=7 \mathrm{~Hz}, 2 \mathrm{H} ; \mathrm{CH}_{2}$ ), 2.70 (m, 4H; $\left.\mathrm{CH}_{2}\right), 3.73$ (s, 2H; $\left.\mathrm{CH}_{2}\right), 5.96\left(\mathrm{~s}, 2 \mathrm{H} ; \mathrm{CH}_{2}\right), 6.80(\mathrm{~s}, 2 \mathrm{H} ; \mathrm{CH}), 6.88(\mathrm{~s}, 1 \mathrm{H} ; \mathrm{CH})$, 7.22-7.33 (m, 5H; CH); ${ }^{13} \mathrm{C}$ NMR $\left(75 \mathrm{MHz}, \mathrm{CDCl}_{3}, 25^{\circ} \mathrm{C}, \mathrm{TMS}\right): \delta=31.6\left(\mathrm{CH}_{2}\right), 33.6$ $\left(\mathrm{CH}_{2}\right), 48.6\left(\mathrm{CH}_{2}\right), 53.7\left(\mathrm{CH}_{2}\right), 100.9\left(\mathrm{CH}_{2}\right), 108.1(\mathrm{CH}), 108.8(\mathrm{CH}), 121.3(\mathrm{CH}), 125.8$ $(\mathrm{CH}), 128.4(\mathrm{CH}), 128.5(\mathrm{CH}), 128.5(\mathrm{CH}), 134.0(\mathrm{Cq}), 142.1(\mathrm{Cq}), 146.6(\mathrm{Cq}), 147.8(\mathrm{Cq})$; MS (ES+) $m / z 270\left(\mathrm{MH}^{+}\right)$; Anal. Cacld for $\mathrm{C}_{17} \mathrm{H}_{19} \mathrm{NO}_{2}$ : C, 75.84; H, 7.06; N, 5.20. Found: C, 75.90; H, 7.10; N, 5.23.

$\mathrm{N}$-(naphtylmethyl)phenylpropylamine (entry 5, Table 2). Pale yellow oil. ${ }^{1} \mathrm{H}$ NMR $(300 \mathrm{MHz}$, $\left.\mathrm{CDCl}_{3}, 25^{\circ} \mathrm{C}, \mathrm{TMS}\right): \delta=1.64(\mathrm{~s}, 1 \mathrm{H} ; \mathrm{NH}), 1.93\left(\mathrm{q}, J=7 \mathrm{~Hz}, 2 \mathrm{H} ; \mathrm{CH}_{2}\right), 2.73(\mathrm{t}, J=7 \mathrm{~Hz}$, 2H; $\mathrm{CH}_{2}$ ), 2.83 (t, $\left.J=7 \mathrm{~Hz}, 2 \mathrm{H} ; \mathrm{CH}_{2}\right), 4.27\left(\mathrm{~s}, 2 \mathrm{H} ; \mathrm{CH}_{2}\right), 7.21-7.35(\mathrm{~m}, 5 \mathrm{H} ; \mathrm{CH}), 7.46-7.59$ (m, 4H; CH), 7.81 (dd, $J=8 \mathrm{~Hz}, J=1.5 \mathrm{~Hz}, 1 \mathrm{H} ; \mathrm{CH}), 7.90$ (dd, $J=8 \mathrm{~Hz}, J=1.5 \mathrm{~Hz}, 1 \mathrm{H}$; $\mathrm{CH}), 8.15(\mathrm{~d}, J=8 \mathrm{~Hz}, 1 \mathrm{H} ; \mathrm{CH}) ;{ }^{13} \mathrm{C} \mathrm{NMR}\left(75 \mathrm{MHz}, \mathrm{CDCl}_{3}, 25^{\circ} \mathrm{C}, \mathrm{TMS}\right): \delta=31.7\left(\mathrm{CH}_{2}\right)$, 
$33.7\left(\mathrm{CH}_{2}\right), 49.4\left(\mathrm{CH}_{2}\right), 51.7\left(\mathrm{CH}_{2}\right), 123.7(\mathrm{CH}), 125.4(\mathrm{CH}), 125.7(\mathrm{CH}), 125.8(\mathrm{CH}), 126.1$ $(\mathrm{CH}), 126.2(\mathrm{CH}), 127.8(\mathrm{CH}), 128.4(\mathrm{CH}), 128.5(\mathrm{CH}), 128.8(\mathrm{CH}), 131.8(\mathrm{Cq}), 133.9(\mathrm{Cq})$, $136.0(\mathrm{Cq}), 142.2(\mathrm{Cq})$; MS (ES+) $m / z 276\left(\mathrm{MH}^{+}\right)$; Anal. Cacld for $\mathrm{C}_{20} \mathrm{H}_{21} \mathrm{~N}$ : C, 87.27; H, 7.63; N, 5.09. Found: C, 87.30; H, 7.68; N, 5.10.

N-(cyclopropylmethyl)phenylpropylamine (entry 8, Table 2). Colorless oil. ${ }^{1} \mathrm{H}$ NMR (300 $\left.\mathrm{MHz}, \mathrm{CDCl}_{3}, 25^{\circ} \mathrm{C}, \mathrm{TMS}\right): \delta=0.10\left(\mathrm{q}, J=4.5 \mathrm{~Hz}, 2 \mathrm{H} ; \mathrm{CH}_{2}\right), 0.50\left(\mathrm{~m}, 2 \mathrm{H} ; \mathrm{CH}_{2}\right), 0.95(\mathrm{~m}$, $1 \mathrm{H} ; \mathrm{CH}$ ), 1.43 (s, 1H; NH), 1.85 (quintet, $J=7.5 \mathrm{~Hz}, 2 \mathrm{H} ; \mathrm{CH}_{2}$ ), 2.47 (d, $J=7.0 \mathrm{~Hz}, 2 \mathrm{H}$; $\left.\mathrm{CH}_{2}\right), 2.70\left(\mathrm{~m}, 4 \mathrm{H} ; \mathrm{CH}_{2}\right), 7.18-7.33(\mathrm{~m}, 5 \mathrm{H} ; \mathrm{CH}) ;{ }^{13} \mathrm{C} \mathrm{NMR}\left(75 \mathrm{MHz}, \mathrm{CDCl}_{3}, 25^{\circ} \mathrm{C}, \mathrm{TMS}\right)$ : $\delta=3.5\left(\mathrm{CH}_{2}\right), 10.9(\mathrm{CH}), 31.4\left(\mathrm{CH}_{2}\right), 33.6\left(\mathrm{CH}_{2}\right), 49.2\left(\mathrm{CH}_{2}\right), 54.9\left(\mathrm{CH}_{2}\right), 125.8(\mathrm{CH}), 128.4$ $(\mathrm{CH}), 141.9(\mathrm{Cq})$; MS (ES+) m/z $190\left(\mathrm{MH}^{+}\right)$; Anal. Cacld for $\mathrm{C}_{13} \mathrm{H}_{19} \mathrm{~N}$ : C, 82.54; H, 10.05; N, 7.41. Found: C, 82.50; H, 10.00; N, 7.38.

\section{Results and discussion}

\subsection{Optimization of the $\mathbf{1}_{\mathrm{x} x}$-mediated oxidation-imine formation-reduction sequence}

At the beginning of our investigation, we focused our attention on the $\mathbf{1}_{\mathbf{o x}}$-mediated catalytic oxidation of an activated primary amine such as benzylamine, in the presence of an unactivated primary amine such as aminomethylcyclopropane. Then, we thought that benzylamine would be engaged in the catalytic process prior to aminomethylcyclopropane which would serve as the alkylating agent (Scheme 2). The $\mathbf{1}_{\mathbf{o x}}$-mediated catalytic oxidation step was realized under the previously reported experimental conditions, which required a platinum anode and methanol as the solvent [21]. Furthermore, a combination of $2.50 \mathrm{mmol}$ of benzylamine with $3.75 \mathrm{mmol}$ of aminomethylcyclopropane and $0.10 \mathrm{mmol}$ of $\mathbf{1}_{\text {red, }}$, which corresponded to $4 \mathrm{~mol} \%$ (relative to benzylamine) of the electrocatalyst $\mathbf{1}_{\mathbf{o x}}$, was found to be ideal for this reaction.

Under the optimized conditions, the cyclic voltammogram of compound $\mathbf{1}_{\text {red }}(0.4$ $\mathrm{mM}$ ), in deaerated $\mathrm{MeOH}$ showed an oxidation peak at $+0.5 \mathrm{~V}$ vs. SCE, due to a two- 
electron process, the sweep rate being $0.1 \mathrm{Vs}^{-1}$. The addition of benzylamine $(10 \mathrm{mM})$ and aminomethylcyclopropane $(15 \mathrm{mM})$ had two effects: first, a shift of the anodic peak to $0 \mathrm{~V}$ vs. SCE as a result of the ionization of the 4-hydroxyl group; second, a slight increase in the anodic peak intensity, which suggested that 3,4-iminoquinone $\mathbf{1}_{\mathbf{0 x}}$ was protected from its subsequent polymerization reaction because it could act as a catalyst for the oxidation of amine. Similar effects have already been observed about the catalytic activity of close quinonoid species [42].

When the controlled potential of the Pt anode was fixed at $+0.6 \mathrm{~V}$ vs SCE, the anodic current remained constant, and the current efficiency obtained by the electrolysis for $3 \mathrm{~h}$ was $100 \%$, indicating that no side reaction took place under the experimental conditions used. Note that a high potential value was intentionally chosen, because of the continuous shift of the anodic peak observed in the course of the electrolysis until $+0.5 \mathrm{~V}$ vs. SCE, when the amine concentration was no longer sufficient to ionize the 4-hydroxyl group of $\mathbf{1}_{\text {red. }}$ These results indicated that the $\mathbf{1}_{\text {red }} / \mathbf{1}_{\mathbf{o x}}$ system behaved as a redox mediator for the indirect electrochemical oxidation of benzylamine to the corresponding $N$-benzylidene cyclopropylmethylamine (Scheme 2). The in situ presence of $N$-benzylidene cyclopropylmethylamine could be evidenced through the UV-vis changes observed in the course of the catalytic anodic process (Figure 1a). After application of the anodic potential, a UV absorption band at $250 \mathrm{~nm}$ developed which was similar to that recorded from a solution of commercially available related $N$-benzylidenemethylamine in methanol. After exhaustive oxidation, the Pt anode was replaced by a mercury pool, because $N$-benzylidene cyclopropylmethylamine could not be reduced at the platinum cathode in methanol. When the potential of the mercury pool was fixed at $-1.6 \mathrm{~V}$ vs SCE, which is at a potential for which $N$ benzylidenealkylamine could be reduced to the corresponding secondary amine, the UV-vis absorption band at $250 \mathrm{~nm}$ disappeared in agreement with the formation of the expected 
secondary amine. Note the UV-vis absorption changes observed in the course of the imine reduction step (Figure 1b) were nearly superimposable upon those observed during the oxidation-imine formation step (Figure 1a). Finally, the two-electron reduction process led to $N$-benzyl- $N$-cyclopropylmethylamine in $80 \%$ yield (entry 1 , Table 1 ). In a control experiment, the chemoselectivity of the oxidation-imine formation reaction was verified by converting the unstable in situ generated alkylimine to the 2,4-dinitrophenylhydrazone (DNPH) by aqueous acidic work-up of the oxidized solution with 2,4-dinitrophenylhydrazine [21]. As expected, no alkylidenebenzylamine was evidenced.

\subsection{Electrocatalyzed $N$-alkylation of benzylamine with diverse alkylating amines}

Having established a reliable set of conditions, we examined the scope of the process using different alkylating amines. The results are summarized in Table 1. Various aliphatic primary amines reacted with benzylamine to give the desired secondary amines $\mathbf{A}$, in moderate to good yields ranging from 57 to $80 \%$. In some cases (entries 2, 8 and 9, Table 1), diamines $\mathbf{B}$ arising from a one-electron reduction mechanism were also observed as dimeric by-products (Scheme 3). The formation of related diamines has been previously reported from the one-electron reduction of aldimines [43-46]. In some cases, the electrochemical method enabled to perform the synthesis of vicinal diamines with a high degree of selectivity $[44,46]$. To minimize the formation of diamines $\mathbf{B}$, which were produced after a bimolecular dimerization reaction, the benzylamine initial concentration did not exceed $10 \mathrm{mM}$. Furthermore, the application of a sufficiently negative working potential (-1.6 V vs SCE) favored the electroreduction of the alkylimine intermediate to the desired amine $\mathbf{A}$. Dibenzylamine $\mathbf{C}$ was also observed as a by-product (entries 2-7 and entry 10, Table 1). However, its formation could be limited to 5-10\% through the addition of an excess of alkylating amine (1.5 equiv). Interestingly, in the case of ethanolamine, the presence of the 
alcohol group did not interfere with the catalytic anodic process, indicating a good tolerance of this functional group (entry 10, Table 1). As a consequence, this one-pot oxidation-imine formation-reduction sequence could be very probably extended to the synthesis of $\beta$ aminoalcohol derivatives.

\subsection{Electrocatalyzed $N$-alkylation of diverse primary amines with phenylpropylamine}

In a second series of experiments, we explored the electrocatalyzed $\mathrm{N}$-alkylation of various amine substrates with phenylpropylamine chosen as the alkylating agent (Table 2). As expected, activated substituted benzylamines were good substrates for this reaction, and the product yield did not markedly depend on the substitution of the phenyl ring (entries 1-4, Table 2). However, in the case of 1-naphtalenemethylamine, the yield of the desired secondary amine A decreased to $51 \%$, while that of bis-naphtalen-2-yl-methylamine $\mathbf{C}$ increased to $25 \%$ (entry 5, Table 2 and Scheme 4). Probably, the stability of the symmetrical alkylimine species facilitated its extrusion during the $\mathbf{1}_{\mathbf{o x}}$-mediated catalytic process at the expense of the unsymmetrical alkylimine. Less activated pyridinemethanamines could be also $\mathrm{N}$-alkylated with phenylpropylamine by the present biomimetic electrocatalytic system (entries 6 and 7, Table 2), whereas non-activated aminomethylcyclopropane gave a lower yield than that expected on the basis of the high current efficiency ( $95 \%$ for $3 \mathrm{~h}$ ), as a result of the lower stability of the produced secondary alkylamine during work-up (entry 8 , Table 2). However, the moderate yield obtained in this case (48\%) is redeemed by the simplicity of our approach, as it would be likely more difficult to access it by conventional chemical methods. 


\section{Conclusion}

In summary, we have developed a novel in situ one-pot oxidation-imine formationreduction route to secondary amines. The key step of the process consists of the $\mathbf{1}_{\mathbf{o x}}$-mediated catalytic oxidation of a primary aliphatic amine substrate, in the presence of a second amine used as the alkylating agent. The secondary generated alkylimine can be then electrochemically reduced, affording the desired secondary amine. The undeniable benefits of this sequence include economy of both atoms and waste generation, leaving ammonia as the sole by-product. Furthermore, the process allows the in situ generation of unstable alkylimines with a good chemoselectivity, at both ambient temperature and pressure. Finally, we suggest that this electrochemical sequence could be a mild alternative to previously reported syntheses. The extension of this process to the synthesis of various $\beta$-aminoalcohol derivatives is envisioned.

\section{References}

[1] For a review on the synthesis of secondary amines see: R.N. Salvatore, C.H. Yoon, K.W. Jung, Tetrahedron 57 (2001) 7785.

[2] L. Blackburn, R. J. K. Taylor, Org. Lett. 3 (2001) 1637.

[3] H. Kanno, R. J. K. Taylor, Tetrahedron Lett. 43 (2002) 7337.

[4] R. J. K. Taylor, M. Reid, J. Foot, S. A. Raw, Acc. Chem. Res. 38 (2005) 851.

[5] K.-I. Fujita, R. Yamaguchi, Synlett 4 (2005) 560.

[6] A. Tillack, D. Hollmann, D. Michalik, R. Jackstell, M. Beller, Tetrahedron Lett. 47 (2006) 8881. 
[7] D. Hollmann, A. Tillack, D. Michalik, R. Jackstell, M. Beller, Chem. Asian J. 2 (2007) 403.

[8] M. H. S. A. Hamid, J. M. J. Williams, Chem. Commun. (2007) 725.

[9] M. H. S. A. Hamid, P. A. Slatford, J. M. J. Williams, Adv. Synth. Catal. 349 (2007) 1555.

[10] L. U. Nordstrom, R. Madsen, Chem. Commun. (2007) 5034.

[11] K.-I. Fujita, Y. Enoki, R. Yamaguchi, Tetrahedron 64 (2008) 1943.

[12] R. Yamaguchi, S. Kawagoe, C. Asai, K.-I. Fugita Org. Lett. 10 (2008) 181.

[13] A. Pontes da Costa, M. Viciano, M. Sanau, S. Merino, J. Tejeda, E. Peris, B. Royo, Organometallics 27 (2008) 1305.

[14] D. Hollmann, S. Bähn, A. Tillack, M. Beller, Angew. Chem. Int. Ed. 46 (2007) 8291.

[15] D. Hollmann, S. Bähn, A. Tillack, M. Beller, Chem. Commun. (2008) 3199.

[16] D. Hollmann, S. Bähn, A. Tillack, R. Parton, R. Altink, M. Beller, Tetrahedron Lett. 49 (2008) 5742.

[17] A. Adenier, M.M. Chehimi, I. Gallardo, J. Pinson, N. Vilà, Langmuir 20 (2004) 8243.

[18] I. Gallardo, J. Pinson, N. Vilà, J. Phys. Chem. B 110 (2006) 19521.

[19] J.L. Bourdelande, I. Gallardo, G. Guirado, J. Am. Chem. Soc. 129 (2007) 2817.

[20] M. Largeron, A. Neudörffer, M.-B. Fleury, Angew. Chem. Int. Ed. 42 (2003) 1026.

[21] M. Largeron, A. Chiaroni, M.-B. Fleury, Chem. Eur. J. 14 (2008) 996.

[22] D. Xu, A. Chiaroni, M.-B. Fleury, M. Largeron, J. Org. Chem. 71 (2006) 6374.

[23] A.R. Katrizky, K. Yannakopoulou, P. Lue, D. Rasala, L. Urogdi, J. Chem. Soc. Perkin Trans 2 (1989) 225.

[24] S. Harada, N. Kowase, N. Tabuchi, T. Taguchi, Y. Dobashi, A. Dobashi, Y. Hanzawa, Tetrahedron 54 (1998) 753. 
[25] S. C. Shim, Y. G. Kwon, C. H. Doh, B. W. Woo, J. O. Baeg, H. S. Kim, T. J. Kim, D. H. Lee, Y. W. Kwak, J. S. Cha, H. S. Lee, J. K. Uhm, Y. B. Park, Bull Korean Chem. Soc. $11(1990) 140$.

[26] L. Blackburn, R. J. K. Taylor, Org. Lett. 3 (2001) 1637. (see the supporting information).

[27] Z. Zhang, L. L. Schafer, Org. Lett. 5 (2003) 4733. (see the supporting information).

[28] D. S. Matteson, G. Y. Kim, Org. Lett. 4 (2002) 2153. (see the supporting information).

[29] C. L. Bumgardner, E. L. Lawton, J. G. Carver, J. Org. Chem. 37 (1972) 407.

[30] P. Mangeney, T. Tejero, A. Alexakis, F. Grosjean, J. Normant, Synthesis (1988) 255.

[31] M. Boisbrun, L. Jeannin, L. Toupet, J.-Y. Laronze, Eur. J. Org. Chem. (2000) 3051.

[32] Y. L. Janin, A.-M. Aubertin, A. Chiaroni, C. Riche, C. Monneret, E. Bisagni, D. S. Grierson, Tetrahedron 52 (1996) 15157.

[33] C. Anastasi, O. Hantz, E. De Clercq, C. Pannecouque, P. Clayette, N. DereuddreBosquet, D. Dormont, F. Gondois-Rey, I. Hirsch, J.-L. Kraus, J. Med. Chem. 47 (2004) 1183.

[34] W. Kurosawa, T. Kan, T. Fukuyama, Org. Synth. 79 (2002) 186.

[35] B. Miriyala, S. Bhattacharyya, J.S. Williamson, Tetrahedron 60 (2004) 1463.

[36] A. Beeby, D. Parker, J.A. Gareth Williams, J. Chem. Soc. Perkin Trans 28 (1996) 1565.

[37] N. S. Chandrakumar, P. K. Yonan, A. Stapelfeld, M. Savage, E. Rorbacher, P. C. Contreras, D. Hammond, J. Med. Chem. 35 (1992) 223.

[38] H. Duerr, K. Zengerle, H. P. Trierweiler, Z. Naturforsch. 43 (1988) 361.

[39] N. D. Cosford, J.-M. Vernier (Sibia Neurosciences, Inc., USA), WO Patent Appl. $9631475,1996$.

[40] J. E. Nordlander, M. J. Payne, M. A. Balk, J. L. Gress, F. D. Harris, J. S. Lane, R. F. Lewe, S. E. Marshall, D. Nagy, D. J. Rachlin, J. Org. Chem. 49 (1984) 133.

[41] J. M. McGill, Synthesis (1993) 1089. 
[42] K. Kano, N. Nakagawa, K. Takagi, T. Ikeda, J. Chem. Soc. Perkin Trans 2 (1997) 1111.

[43] L. Horner, D.H. Skaletz, Liebigs Ann. Chem. (1975) 1210.

[44] H. Tanaka, T. Nakahara, H. Dhimane, S. Torii, Synlett (1989) 51.

[45] M. Largeron, M.-B. Fleury, J. Org. Chem. 65 (2000) 8874.

[46] T. Siu, W. Li, A. K. Yudin, J. Comb. Chem. 3 (2001) 554.

\section{Acknowledgments}

The author thanks Dr. M.-B. Fleury, Emeritus Professor at the University of Paris 5, for helpful discussions. 


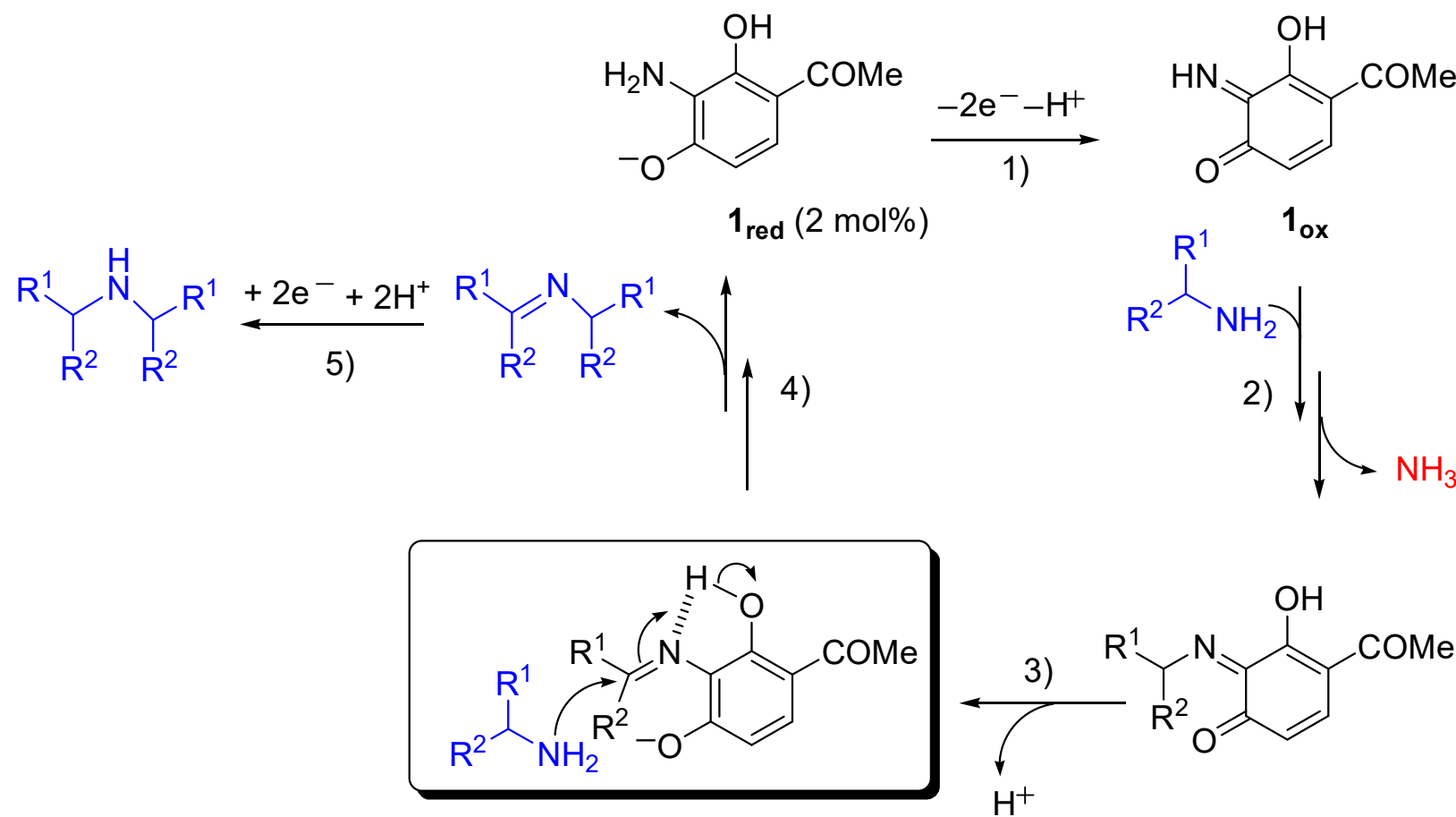

Scheme 1. One-pot $\mathbf{1}_{\mathbf{o x}}$-mediated oxidation-imine formation-reduction route to secondary amines from primary amines. 


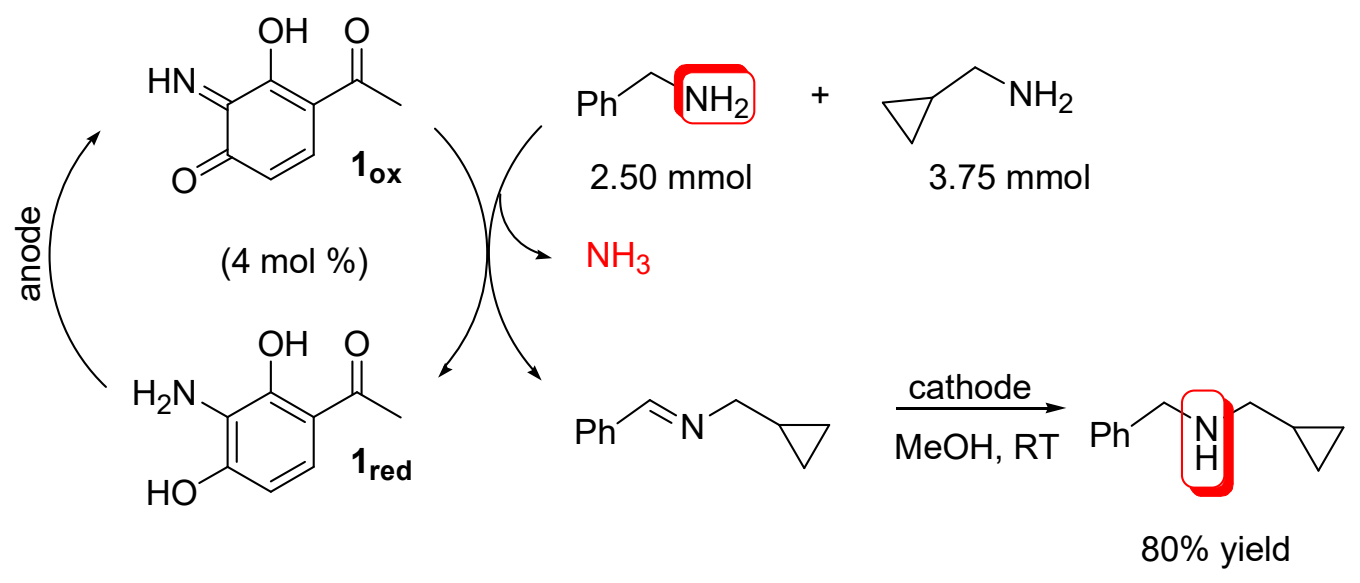

Scheme 2: Chemoselective electrocatalyzed N-alkylation of benzylamine with aminomethylcyclopropane. 


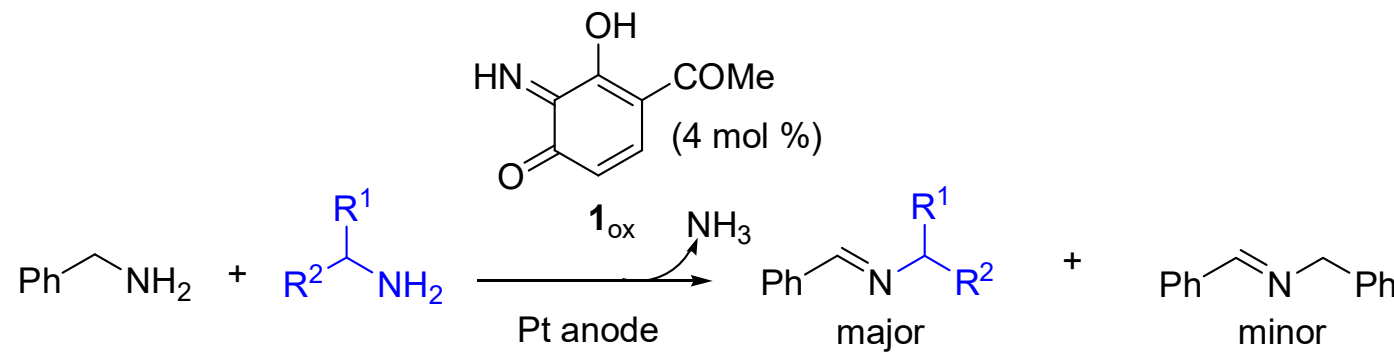

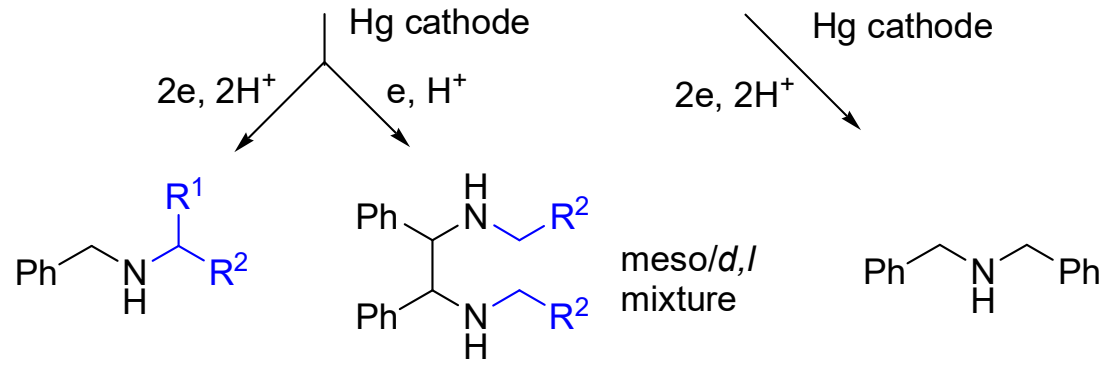

A $\quad$ B $\mathrm{R}^{1}=\mathrm{H} \quad$ C

Scheme 3: Electrocatalyzed N-alkylation of benzylamine with different primary amines 


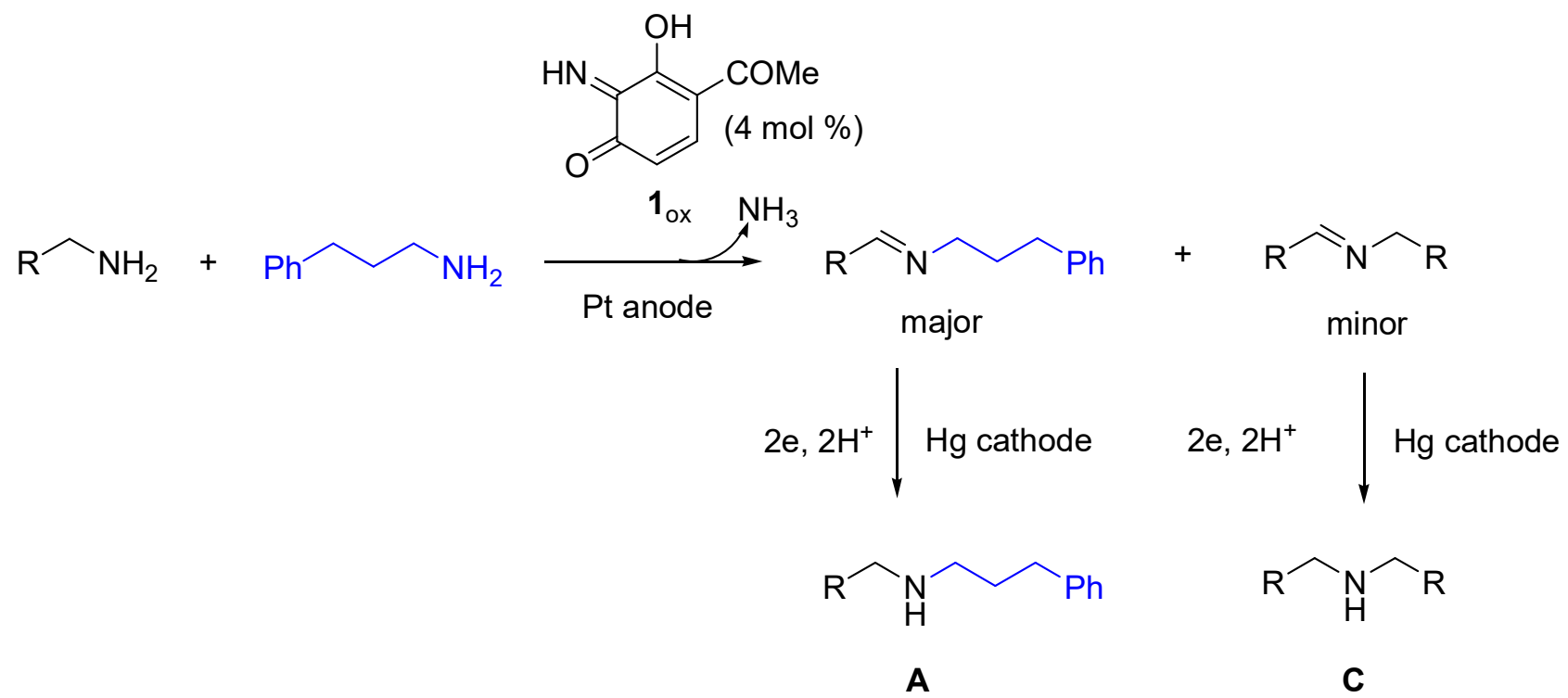

Scheme 4: Electrocatalyzed N-alkylation of various amine substrates with phenylpropylamine. 


\section{Caption to figure}

Figure 1. Spectrophotometric changes accompanying the $\mathbf{1}_{\mathbf{o x}}$-mediated oxidation-imine formation-reduction sequence for the N-alkylation of benzylamine (2mM) with aminomethylcyclopropane $(3 \mathrm{mM})$ in $\mathrm{MeOH}$ : a) oxidation-imine formation step; b) imine reduction step. Spectra recorded: before anodic (a) or cathodic (b) controlled potential electrolysis as well as after exchange of $0.5,1.0,1.5$ and 2 mol of electrons (relative to benzylamine), respectively. Cell thickness is $0.1 \mathrm{~cm}$. 


\section{Table 1}

Electrocatalyzed $\mathrm{N}$-alkylation of benzylamine with different primary amines. Yield of isolated products $^{\mathrm{a}}$

\begin{tabular}{|c|c|c|c|c|}
\hline Entry & $\begin{array}{l}\text { Alkylating } \\
\text { Amine }\end{array}$ & $\begin{array}{l}\text { Amine product } \mathbf{A} \\
\text { Yield }^{\mathrm{b}}(\%)\end{array}$ & $\begin{array}{l}\text { Diamine B } \\
\text { Yield }^{\mathrm{b}}(\%)[\mathrm{meso} / d, l]\end{array}$ & $\begin{array}{l}\text { Dibenzylamine C } \\
\text { Yield }^{\mathrm{b}}(\%)\end{array}$ \\
\hline 1 & & 80 & - & - \\
\hline 2 & & 60 & $15[65 / 35]$ & 5 \\
\hline 3 & & 68 & - & 10 \\
\hline 4 & & 66 & - & 10 \\
\hline 5 & & 70 & - & 10 \\
\hline 6 & & 57 & - & 12 \\
\hline 7 & & 64 & - & 8 \\
\hline 8 & $\mathrm{MeC}$ & 71 & 5 [70/30] & - \\
\hline 9 & & 60 & $15[75 / 25]$ & - \\
\hline 10 & $\mathrm{HO}$ & 68 & - & 10 \\
\hline
\end{tabular}

${ }^{\text {a }}$ Reagents and conditions: (benzylamine) $=10 \mathrm{mM}$, (alkylating amine) $=15 \mathrm{mM},\left(\mathbf{1}_{\mathrm{ox}}\right)=0.4$ $\mathrm{mM}$ (4 mol \% relative to benzylamine), $\mathrm{MeOH}, \mathrm{RT}$. Oxidation-imine formation step: $\mathrm{Pt}$ anode $(\mathrm{E}=+0.6 \mathrm{~V}$ vs SCE$)$, 3h. Imine reduction step: $\mathrm{Hg}$ cathode $(\mathrm{E}=-1.6 \mathrm{~V}$ vs SCE$), 1 \mathrm{~h} .{ }^{\mathrm{b}}$ Yields refer to chromatographically pure isolated products and are relative to benzylamine. 
Table 2

Electrocatalyzed N-alkylation of various amine substrates with phenylpropylamine. Yield of isolated products ${ }^{\mathrm{a}}$

\begin{tabular}{lll}
\hline Entry & $\begin{array}{l}\text { Amine product } \mathrm{A} \\
\text { Yield }^{\mathrm{b}}(\%)\end{array}$ & $\begin{array}{l}\text { Amine by-product } \mathbf{C} \\
\text { Yield }\end{array}$ \\
\hline Substrate
\end{tabular}

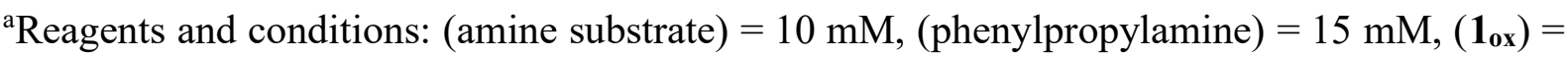
$0.4 \mathrm{mM}$ (4 mol \% relative to the amine substrate), $\mathrm{MeOH}, \mathrm{RT}$. Oxidation-imine formation step: Pt anode ( $\mathrm{E}=+0.6 \mathrm{~V}$ vs $\mathrm{SCE}), 3 \mathrm{~h}$. Reduction step: $\mathrm{Hg}$ cathode $(\mathrm{E}=-1.6 \mathrm{~V}$ vs $\mathrm{SCE})$, 1h. ${ }^{\text {b}}$ Yields refer to chromatographically pure isolated products and are relative to the amine substrate. 
a)

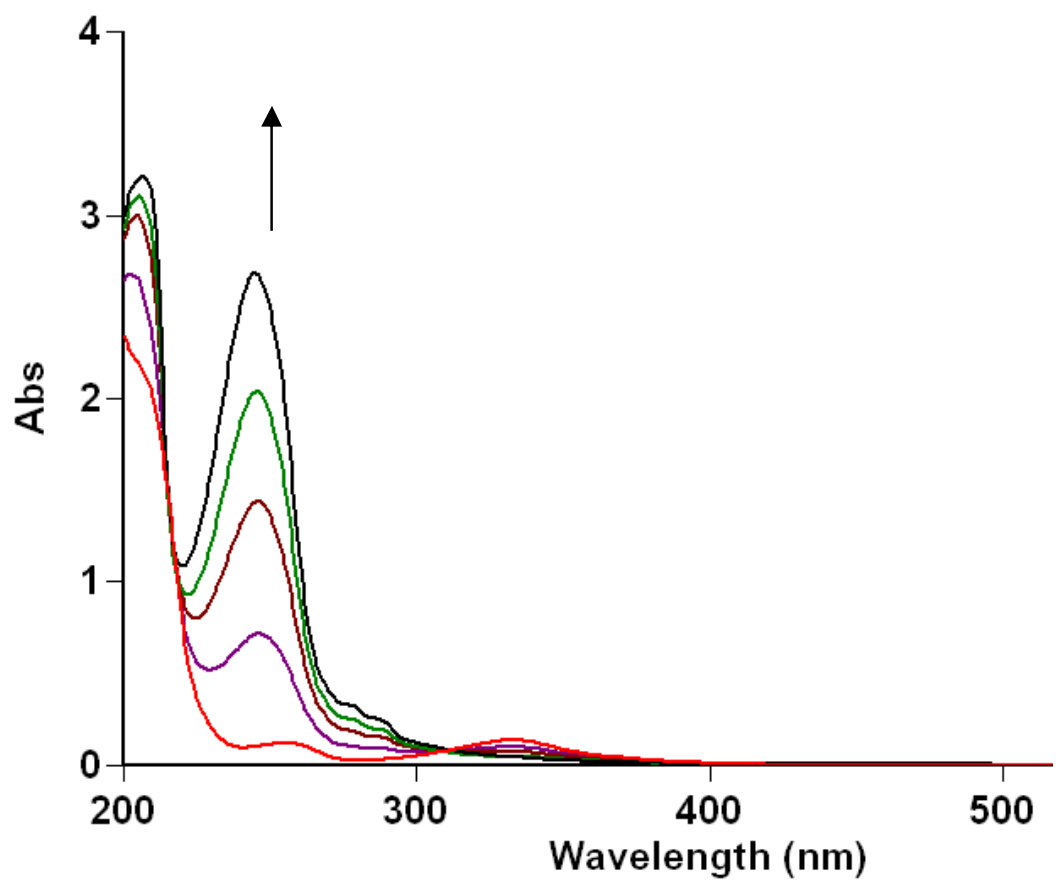

b)

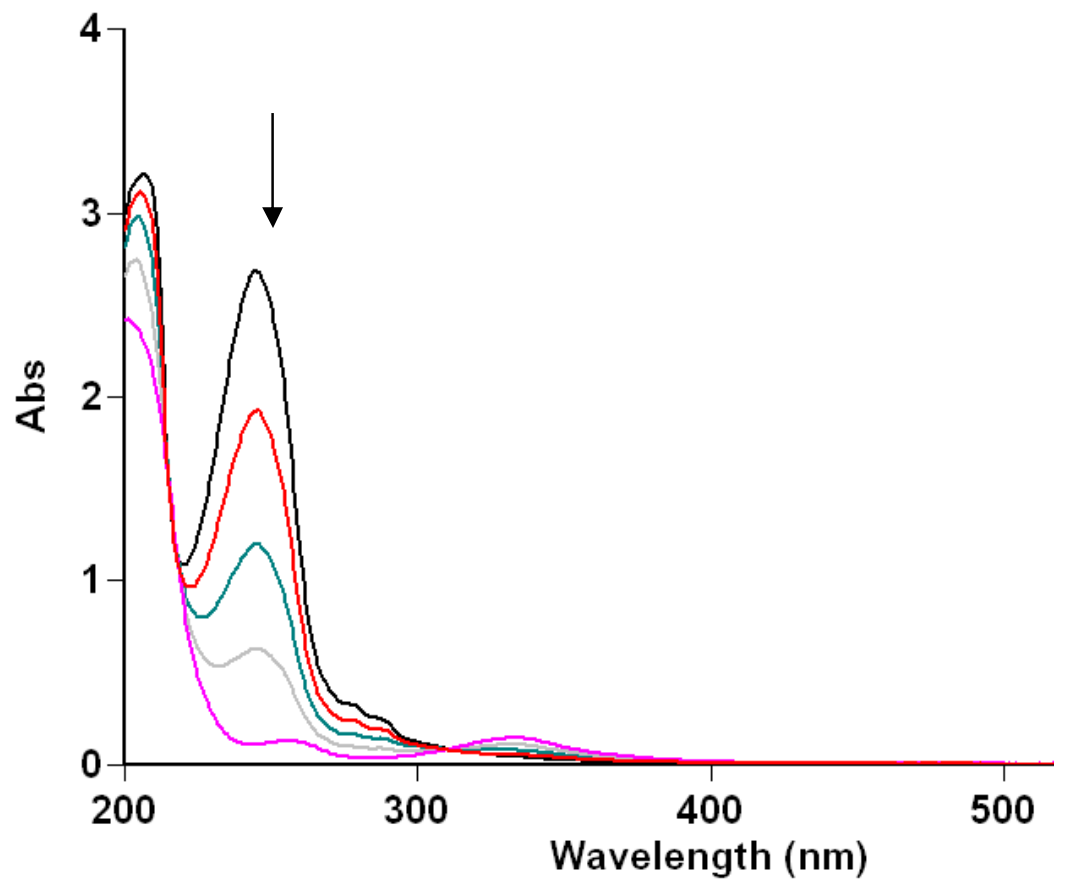

Figure 1 